\section{Bilder von höchster Qualität}

Intraoralröntgen mit Sensoren ist bei richtiger Handhabung und Positionierung unkompliziert. Zahnfilm und Speicherfolie sind vergleichbar in ihrer praktischen Anwendung. Röntgensensoren unterscheiden sich von diesen bekannten Röntgentechniken nur durch ihre Positionierung direkt zentral in der Mundhöhle. Es entstehen dadurch Aufnahmen von herausragender Qualität in nur wenigen Sekunden. Mit Sabine Hiemer, Qualitätsmanagerin/Auditorin und externe Systemberaterin der Firma Dentsply Sirona zur Begleitung von Zahnarztpraxen während der Neueinführung intraoraler Röntgensensoren, sprach Eva Maria Leßmann.

? Frau Hiemer, Sie beraten und trainieren Praxisteams im Umgang mit digitalen Röntgensensoren. Wie reagieren diese auf neue Technik?

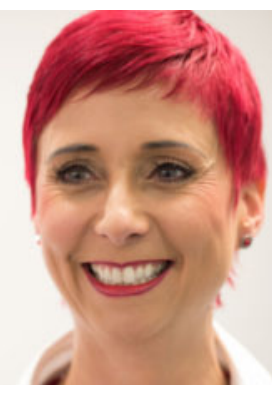

S. Hiemer: Grundsätzlich gibt es in den Praxen keine Vorbehalte. Der Umgang mit neuer Technik bedarf immer einer bestimmten Eingewöhnungszeit und einer Anpassung der bisherigen Arbeitsabläufe. Der Wunsch der Anwender nach Innovation und Optimierung der Arbeitsprozesse lässt anfängliche Startschwierigkeiten durch die Größe des Sensors und die neuen Positionierungsmöglichkeiten im Training schnell überwinden. Überzeugend ist auch der einfache Kabelwechsel bei Bedarf.

? Wie steht es um den Komfort des Röntgensensors?

Im Vergleich zur Speicherfolie oder dem Zahnfilm ist der XIOS XG Supreme breiter und starr, nicht flexibel. Der entscheidende Unterschied beim Röntgen mit dem Sensor ist die neue Positionierung zentral in der Mitte der Mundhöhle, nicht an der Zahnreihe, wie bisher gewohnt. Er wird zwischen dem Mundboden, der Zunge und dem Gaumendach fixiert. Die abgerundeten Seitenflächen vergrößern die Auflagefläche, es gibt keine scharfen Kanten wie beim Film oder der Speicherfolie, dadurch empfindet der Patient den Sensor als angenehm und sehr komfortabel.

? Warum wird der Sensor anders platziert als eine Speicherfolie?

Für eine rechtssichere Diagnostik benötigen Zahnärztin oder Zahnarzt reproduzierbare orthoradiale Röntgenaufnahmen, auf denen alle Strukturen 1:1 unverzerrt abgebildet sind. Diese entstehen bei richtiger Anwendung der Parallel-Röntgentechnik mit geeignetem Haltersystem. Bei den Röntgenaufnahmen mit Film oder Speicherfolie sind durch anatomische Besonder- heiten im Seiten- und Frontzahnbereich überwiegend Aufnahmen mit Halbwinkeltechnik möglich, dadurch werden nicht immer realistische Darstellungen des Zahns erzielt. Bei schmalem Kiefer, hohem oder flachem Gaumen sowie flachen Mundböden entstehen mit Film oder Speicherfolie häufig leichte Verzerrungen der mesialen und distalen Zahnwurzeln im Seitenzahnbereich bzw. werden Frontzähne verlängert oder verkürzt dargestellt. Bei richtiger Positionierung der Sensoren ist einen bessere Bildqualität zu erzielen.

? Und zusätzlich gibt es mit dem XIOS XG Supreme noch die Möglichkeit der Bildbearbeitung.

Der Supreme-Sensor besitzt eine theoretische Auflösung von 33 Linienpaaren/mm und erzielt damit eine herausragende Bildqualität. Behandelnde können durch Nutzung des dynamischen Schärfereglers die Aufnahme in Bezug aufSchärfe, Helligkeit und der gewünschten Kontraste zusätzlich bearbeiten und damit in der Diagnostik nach den individuellen Wünschen optimieren.

\section{? Wo sind Sensoren darüber hinaus überlegen?}

Sensoren überzeugen beim Zeitmanagement und der Wirtschaftlichkeit. Es entfallen Entwicklungs- bzw. Scangerät, der Scanprozess sowie die Umwandlungszeit der Speicherfolie und Zahnfilme. Beim Sensor steht den Behandelnden das Röntgenbild sofort zur Diagnostik, Auswertung und Planung zur Verfügung. Bei Bedarf, oder wenn es die Indikation erfordert, können zusätzliche Bilder sofort erstellt werden ohne unnötige Wartezeit und weitere Arbeitsprozesse. Chirurgischen Praxen ermöglicht das Röntgen mit dem Sensor während der Operation (z. B. Implantation) ein besseres Zeitmanagement, verkürzt die Operationszeit und verringert damit das Risiko einer Wundheilungsstörung. Der innovative Sensor bringt einen zusätzlichen Wert für die

Praxis: Die Ergebnisse lassen sich gut kommunizieren, was die Beratung der Patienten erleichtert. Darüber hinaus sichert die Darstellung von Gewebe- und Zahnstrukturen mit ausgezeichneter und überzeugender Bildqualität eine rechtssichere Diagnostik und Dokumentation.

\begin{abstract}
? Weshalb ist Ihrer Meinung nach ein Training ratsam, bevor Intraoralsensoren eingesetzt werden?

Jeder Praxis empfehle ich bei Neueinstieg in diese innovative Technik ein persönliches Einzeltraining. So werden Anfängerfehler vermieden, und der Trainer kann direkt auf die individuellen Bedürfnisse der Praxis eingehen. Fehler beim Positionieren und eine daraus folgende Wiederholung von Röntgenaufnahmen werden vermieden.
\end{abstract}

? Welchen Zeitaufwand muss man dafür einplanen?

Das effektivste und interessanteste Training ist immer das direkt im Team in der Zahnarztpraxis. Dafür benötigen wir 3-5 Stunden, je nach Teilnehmerzahl. Basics der Röntgentechnik und Strahlenkunde sind ein guter Einstieg, um die Positionierung des Sensors zu verstehen. Jeder Mitarbeiter erlernt das Handling in der direkten Anwendung des Sensors sehr leicht, jede Position wird einzeln trainiert.

? Fällt die Umstellung den Praxen denn schwer?

Praxisteams sind aufgeschlossen für interessante Innovationen und erlernen das Handling schnell. Sie nutzen das Training auch zum Auffrischen und Festigen bestehender Kenntnisse in Sachen Strahlenschutz. Nach der Übungszeit von 2 Wochen gibt es ein Feedbackgespräch mit der Trainerin. Bestehende Fragen und mögliche Fehler werden dadurch schnell korrigiert.

? Sind die XIOS XG Supreme-Sensoren mit Film- und Folienhaltern kompatibel? Ich rate davon ab, Halter zu verwenden, die nicht speziell für XIOS XG Supreme Sensoren vorgesehen sind. Dentsply Sirona empfiehlt ein passendes Einweghaltersysteme für Risikopatienten und 2 Mehrweghaltersysteme.

Frau Hiemer, vielen Dank für das Gespräch

Dieser Beitrag entstand in Zusammenarbeit mit Dentsply Sirona, A - Wals bei Salzburg 